\title{
Comparison of single-use and reusable flexible ureteroscope for renal stone management: a pooled analysis of 772 patients
}

\author{
Yongchao Li^^${ }^{\#}$, Jinbo Chen", Zewu Zhu, Huimin Zeng, Feng Zeng, Zhiyong Chen, Zhongqing Yang, \\ Yu Cui, Hequn Chen, Yang Li^ \\ Department of Urology, Xiangya Hospital, Central South University, Changsha, China \\ Contributions: (I) Conception and design: Y Li, J Chen; (II) Administrative support: Z Zhu, Z Chen, H Chen, Y Li; (III) Provision of study materials \\ or patients: F Zeng, Y Cui; (IV) Collection and assembly of data: Y Li, J Chen; (V) Data analysis and interpretation: H Zeng, Z Yang; (VI) \\ Manuscript writing: All authors; (VII) Final approval of manuscript: All authors. \\ "These authors contributed equally to this work as co-first authors. \\ Correspondence to: Yang Li. Department of Urology, Xiangya Hospital, Central South University, Changsha 410008, China. Email: liyang7365@163.com.
}

\begin{abstract}
Flexible ureteroscopy is a common therapy for patients with renal calculi. In recent years, the prevalence of single-use flexible ureteroscope (FURS) use has been on the rise. Thus, several trials have been conducted to compare the efficacy between single-use and reusable FURS. The aim of this meta-analysis was to systematically assess the effectiveness and safety of single-use $v s$. reusable FURS in treating renal stones. PubMed, Web of Science, Cochrane Library and EMBASE were researched to identify relevant studies up to September 2019. Article selection was performed through the search strategy based on Preferred Reporting Items for Systematic Reviews and Meta-Analyses criteria. The Newcastle-Ottawa Scale was applied to assess the methodological quality of non-randomized controlled trials, and the methodological quality of randomized controlled trials was evaluated using the Jadad scale. A total of five studies with 772 patients were included in the meta-analysis, including two randomized controlled trials, two single-centre prospective studies, and one prospective case-control trial. The pooled results showed that single-use FURS was associated with a higher stone-free rate (SFR) (OR: 1.50; 95\% CI, 1.06-2.12; P=0.02) than reusable FURS. A significant difference was noted in operative time, and single-use FURS was associated with a longer operative duration (MD: $7.39 \mathrm{~min}$; 95\% CI, 1.75-13.03; $\mathrm{P}=0.01$ ). No significant difference was noted in perioperative complications (OR: 0.97; 95\% CI, 0.56-1.70; $\mathrm{P}=0.92$ ). Subgroup analysis showed no significant difference in urinary tract infection (OR: 0.80; 95\% CI, 0.44-1.46; P=0.46), stent migration (OR: 0.56; 95\% CI, 0.19-1.65; $\mathrm{P}=0.30$ ) or acute kidney injury (OR: 0.76; 95\% CI, 0.16-3.57; P=0.73). Single-use FURS is an effective and safe alternative to reusable FURS for the management of renal stones.
\end{abstract}

Keywords: Renal stones; single-use flexible ureteroscope; reusable flexible ureteroscope

Submitted Jun 23, 2020. Accepted for publication Nov 10, 2020.

doi: $10.21037 /$ tau-20-1009

View this article at: http://dx.doi.org/10.21037/tau-20-1009

\section{Introduction}

Urinary stone disease, or urolithiasis, is one of the most common diseases in the urology field and affects approximately $12 \%$ of the world's population at some stage in their lifetime (1). Transformation of lifestyle factors, such as decreases in physical activity, has increased the prevalence of urolithiasis in recent decades (2). Widely used therapy procedures include extracorporeal shockwave lithotripsy (SWL), percutaneous nephrolithotomy (PCNL) and flexible

^ ORCID: Yang Li, 0000-0002-3993-1537; Yongchao Li, 0000-0002-3993-1537. 
ureteroscope (FURS) $(3,4)$. Studies worldwide have shown an increasing trend for FURS in the treatment of renal stones (5). With technical and optical improvements, FURS has become a more widespread approach for intrarenal stone removal. Furthermore, in some countries, FURS is used as a first-line treatment modality for renal stones $<20 \mathrm{~mm}$ (4).

The first record of the traditional FURS was provided by Marshall in 1964 (6). It is mostly used for the treatment of upper urinary stones $<20 \mathrm{~mm}$ in size and has stone-free rates (SFRs) up to $90 \%$ (7). Although traditional reusable FURS is less invasive has less blood loss and shorter hospital stays compared with PCNL (8), some constraints remain, including a high initial purchase cost, high maintenance costs, and a risk of cross-infection, which resulted in the development of single-use FURS $(9,10)$.

When Bagley pioneered the disposable FURS for accessing the upper urinary tracts in 1987 (11), it was only used to monitor fragments after lithotripsy and diagnose anatomical abnormalities of the upper urinary tract. Over the last few years, there have been encouraging results on the improvement of single-use FURS. The results of many in vitro trials of single-use FURS were comparable to those of reusable FURS in terms of optics, deflection, and irrigation flow $(9,12,13)$.

Previous studies have indicated that reusable FURS rated highly in terms of manoeuvrability and image quality $(14,15)$. Clinical studies have indicated that single-use digital FURS performed comparably to current reusable FURS in terms of the SFR, operative time and complication rate (16). Hence, we performed a meta-analysis to assess the effectiveness and safety of single-use FURS versus reusable FURS in the treatment of stone disease in patients. We present the following article in accordance with the PRISMA reporting checklist (available at: http://dx.doi. org/10.21037/tau-20-1009).

\section{Methods}

\section{Data sources and search}

A literature search was performed for all studies published before September 2019 in the PubMed, Web of Science, Cochrane Library and EMBASE online databases. The search strategy was performed with the following search terms: (flexible ureteroscopes OR retrograde intrarenal surgery OR FURS OR RIRS) AND (single-use flexible ureteroscopes OR disposable flexible ureteroscopes OR single-use FURS OR disposable FURS) AND (renal calculi OR renal stones OR nephrolithiasis OR stone disease). References of original studies were also screened to identify additional reports.

\section{Study selection}

Article selection was performed independently in accordance with the process based on Preferred Reporting Items for Systematic Reviews and Meta-Analyses (PRISMA) guidelines (17). The inclusion criteria for the final selected studies were as follows: (I) original comparative studies reporting at least one of the following outcomes for both single-use FURS and reusable FURS: SFR, operation time, perioperative complications and (II) the full text of the study was available in English. However, studies that published as conference abstracts or posters; studies that failure to provide one of these outcomes of interest; or studies that including paediatric patients ( $<18$ years old) were excluded.

\section{Quality assessment}

According to the widely used criteria provided by the Oxford Centre for Evidence-Based Medicine, the methodological quality of the non-randomized retrospective case-control studies was assessed using the modified Newcastle-Ottawa Scale (NOS), which assesses a maximum of 9 points for the lowest risk of bias in 3 domains: (I) selection; (II) comparability; and (III) exposure and outcomes. A study was considered "high quality" with $>6$ points and "low quality" with $<3$ points.

The Jadad scale (also known as the Oxford quality scoring system) was applied to evaluate the methodological quality of RCTs. A study was considered "high-quality" with $>3$ points, and studies with scores $<2$ points were considered "low quality".

\section{Data extraction and statistical analysis}

Two independent investigators extracted the following information for each study: first author's name, year of publication, number of patients (single-use FURS group and reusable FURS group), mean operation time, stonefree rate and complication rate. To extract the data, a standardized Excel file was used. We chose the article with the most recent data when the same trial appeared 
in different publications. Meta-analyses of comparable data were performed with Review Manager Software (RevMan V5.3, Cochrane Collaboration, Oxford, UK). The experimental group was a single-use FURS group, while the control group was a reusable FURS group. For the binary variables, including stone-free rate and incidence of complications, odds ratios (ORs) were employed, and for the continuous parameters, including operative duration, mean differences (MDs) were employed. We used forest plots to express the results of the meta-analysis. Heterogeneity among the included studies was assessed with a chi-square test and $\mathrm{I}^{2}$ statistic, in which $\mathrm{I}^{2}>50 \%$ or $\mathrm{P}<0.10$ was considered statistically significant heterogeneity. Where heterogeneity among studies was not detected, pooled estimates were calculated with a fixed-effect model (Mantel-Haenszel method). When there was evidence of heterogeneity, a random-effect model (DerSimonian-Laird method) was used. To explore the reliability of the results, sensitivity analysis was applied by omitting a specific study each time. To assess publication bias, a funnel plot was routinely performed.

\section{Results}

\section{Search results and study characteristics}

As illustrated in Figure 1, 128 studies were initially identified, and we excluded 123 articles, including one cadaveric feasibility study, one porcine feasibility study and forty-three irrelevant studies. Five studies were excluded because they were cost comparison analyses, and five studies were excluded because they compared mechanical, optical and irrigation properties. Six-eight studies were excluded because they were comments or reviews. We found one additional record identified through reference lists, and a total of five trials were eligible for final inclusion, all of which were full-text articles. In Table 1, we summarize the baseline characteristics of the 5 studies, such as age, sex ratio, body mass index, stone burden, and model of single-use FURS and reusable FURS. In a study by JK, two different models of single-use FURS were available (Disposable 1: Lithovue Disposable 2: PU3022A). The definition of SFR in the included studies was inconsistent, and one study reported that a residual fragment $<2 \mathrm{~mm}$ in diameter was considered an insignificant clinical fragment. The rest of the included studies defined a residual fragment $<4 \mathrm{~mm}$ as an insignificant clinical fragment. CT (computer tomography), BUS (B ultrasound) and KUB (X-ray of the kidney, ureter and bladder) were used to determine the SFR.

\section{Study quality}

According to the NOS, the methodological quality of two single-centre prospective trials and one prospective casecontrol trial were judged to be high, ranging from 6-7 (Table S1), and Jadad scores for 2 RCTs ranged from 5 to 6 (Table S2). High quality was observed among all included studies.

\section{Operative time}

Four included studies provided enough data available on operative time (14,18-20). In Kam's study, two different models of single-use FURS were compared with the same traditional FURS. Significant heterogeneity $\left(\mathrm{I}^{2}=89 \%\right)$ among studies was identified; thus, a random-effect model was used. The results of the meta-analysis indicated that single-use FURS was associated with a significantly longer operative time (MD: $7.39 \mathrm{~min}$; 95\% CI, 1.75-13.03; $\mathrm{P}=0.01$ ) (Figure 2). Since there are two types of single-use FURS: single-use digital FURS and single-use fiberoptic FURS and 3 of 4 studies used the single-use digital FURS, a subgroup analysis was conducted. There is no significant difference between the single-use digital FURS and reusable FURS (MD: $6.32 \mathrm{~min}$; 95\% CI, -1.04 to 13.68 ; $\mathrm{P}=0.09$ ) (Figure 3). In the study by QS, the operative time was defined as the time from ureteroscope insertion until the end of Foley catherization. However, in the study by Ding et al., the operation time was recorded from the insertion of a rigid ureteroscope to the placement of a double $\mathrm{J}$ stent.

\section{SFR}

Four trials with 686 participants described the SFR $(7,18-20)$, and pooled results indicated that compared to reusable FURS, single-use FURS provided a significantly higher SFR (OR: 1.50; 95\% CI, 1.06-2.12; P=0.02) (Figure 4) without detection of statistical heterogeneity $\left(\mathrm{I}^{2}=0 \%\right)$. In the studies by Qi et al. and Ding et al., "stonefree" status was defined as no residual stones or stones $\leq 4 \mathrm{~mm}$, while in Mager's research, stone-free status was assessed intraoperatively by the surgeon's endoscopic and fluoroscopic view. In the study by Usawachintachit et al., residual stone status was classified as stone free (no fragments present) or insignificant residual fragment (residual fragments $<2 \mathrm{~mm}$ ). 


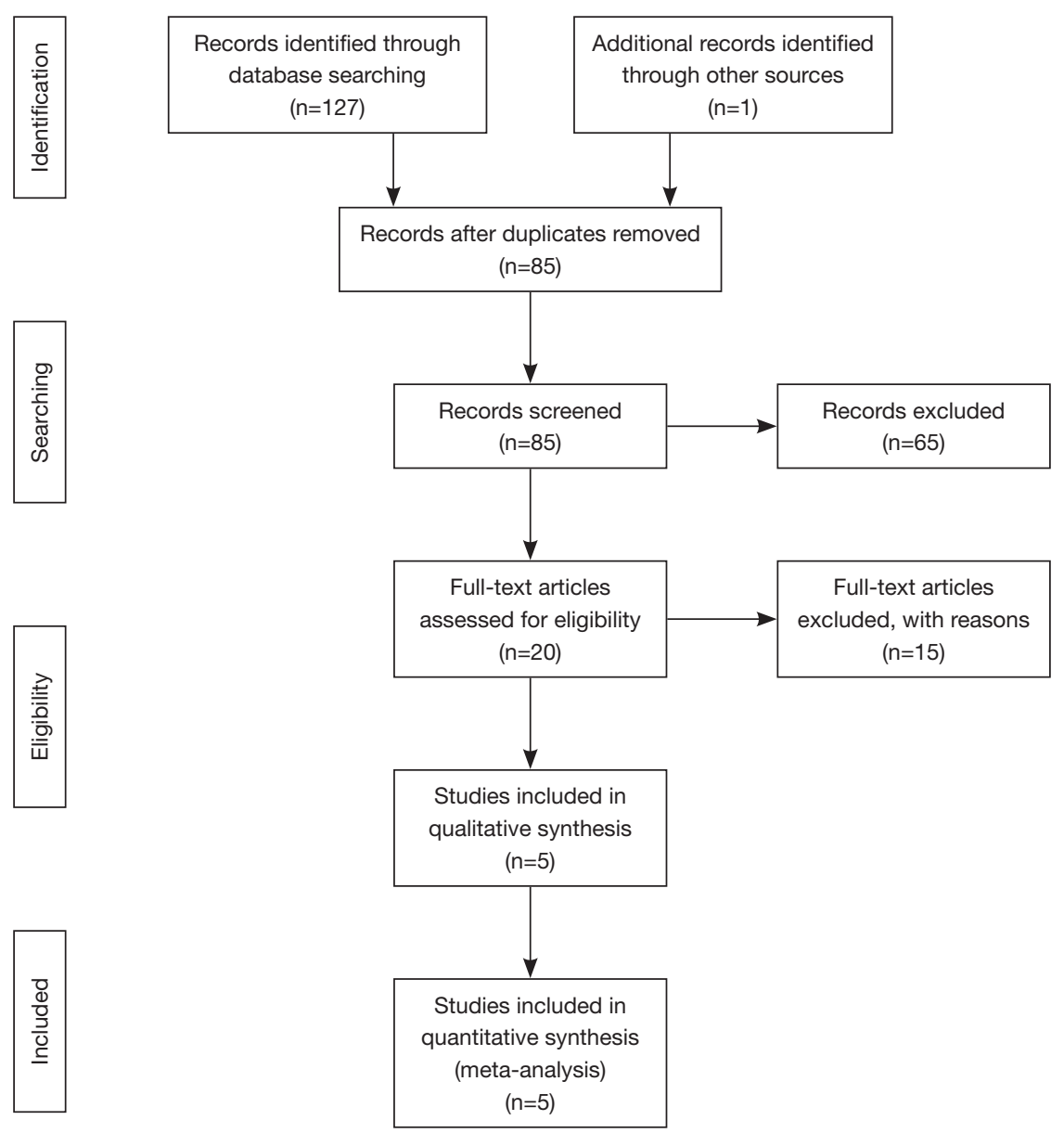

Figure 1 PRISMA flow diagram of study selection.

\section{Perioperative complications}

Four included studies with 842 participants had enough data relevant to perioperative complications $(14,18-20)$, and ORs were used for statistical analysis. Due to the detection of high statistical heterogeneity $\left(\mathrm{I}^{2}=56 \%\right)$, a randomeffect model was used. The pooled results indicated no significant difference between the two groups with respect to perioperative complications (OR: 0.97; 95\% CI, $0.56-$ 1.70; $\mathrm{P}=0.92$ ) (Figure $5 A$ ). In the studies by Kam et al. and Usawachintachit et al., perioperative complications were classified using the Clavien-Dindo classification system. Next, we separately analysed complications. The analysis showed no significant difference between the two groups related to acute renal injury (OR: 0.76; 95\% CI, 0.16-3.57; $\mathrm{P}=0.73$ ) (Figure $5 B$ ), urinary tract infection (OR: 0.80; $95 \%$
CI, 0.44-1.46; $\mathrm{P}=0.46$ ) (Figure 5C) or stent migration (OR: 0.56; 95\% CI, 0.19-1.65; $\mathrm{P}=0.30$ ) (Figure 5D).

\section{Publication bias and sensitivity analyses}

The funnel plot for the SFR are shown in Figure 6. To assess the effect of a single study on the overall estimate, sensitivity analyses were conducted by excluding each study sequentially, one at a time (Figure 7). In our meta-analysis, there were no studies that could influence the summary of risk estimates.

\section{Discussion}

To date, this study is the latest comprehensive meta-analysis to investigate the efficacy of single-use and reusable FURS. 


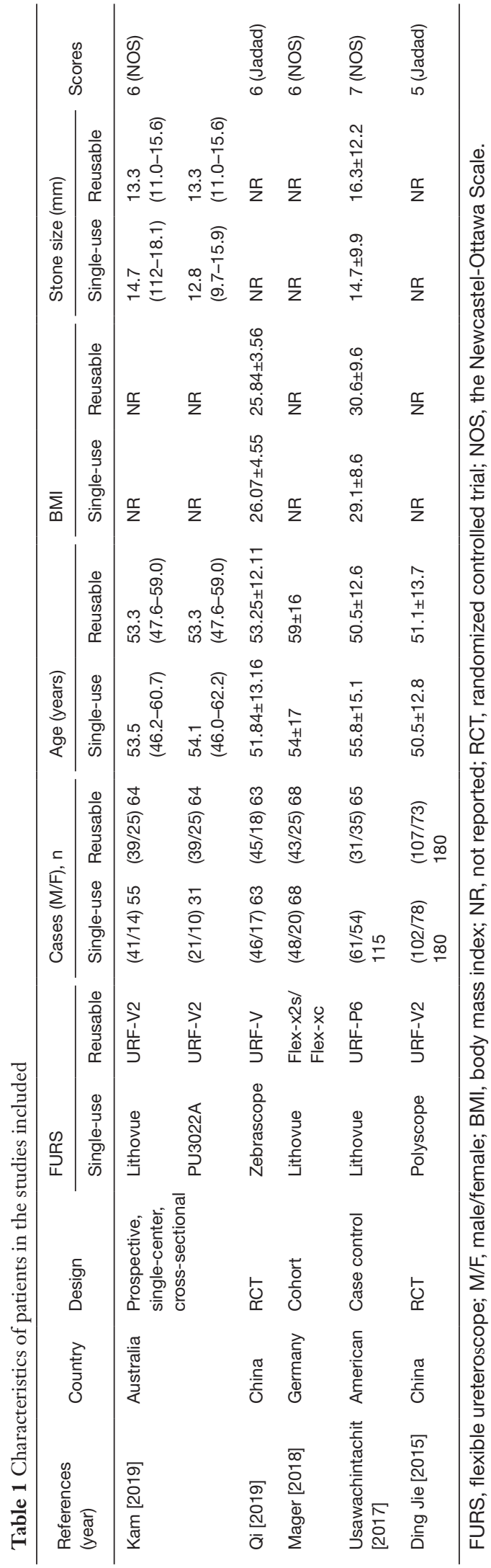

Based on five studies, the results from our meta-analysis suggested that there was no significant difference between the two groups in terms of perioperative complication rate. Single-use FURS was associated with a higher stone free rate but a longer operative time compared with reusable FURS. However, Subgroup analysis indicated that there was no significant difference between the single-use digital FURS and reusable FURS regarding operative time. The potential reason for single-use FURS having a longer operative time may be its lower image quality scores. JK found that the reusable FURS outperformed the singleuse FURS in terms of visibility rate on a 5 -point Likert scale (14), which is consistent with previous researches $(15,21,22)$. As is shown in the Table 1 , the type of singleuse and reusable FURS compared in each study were different. LithoVue, PU3022A, ZebraScope are single-use digital FURS, PolyScope is single-use fiberoptic FURS. Notably, the digital FURS usually perform better than fiberoptic FURS in terms of image quality. The research indicated the mean operative time was significantly longer in the fiberoptic FURS compared with digital FURS (23). Furthermore, the operation of single-use FURS is requires longer training under guidance, a learning curve showed that with the increase in the amount of operations, the mean operative time gradually decreased (18).

A study conducted by Bell et al. compared the LithoVue with URF-P5/P6 and the digital Flex Xc, found that the LithoVue scored lower on most user comfort and maneuverability (21). In the study by Qi et al. (20), the authors found that the nitinol basket usage rate of singleuse FURS was higher than that of reusable FURS. It seems that single-use FURS has superior operability, which is responsible for the higher SFR.

Radical changes have taken place in the field of endourology and stone treatment due to technological innovation during recent decades. FURS has become an effective and safe choice for renal stones due to better access and visualization of the upper urinary tract. Furthermore, since it is less invasive than PCNL, FURS is considered a first-line option for kidney stones, particularly for stones $<20 \mathrm{~mm}$ (4). Even so, this technique still has several disadvantages, including the frequent need for repair, high cost of acquisition and maintenance and high risk of cross infection. As a consequence, the single-use ureteroscope has gained widespread popularity aiming to offer solutions to the possibility of contamination and high cost issues. To date, there are more than 10 different single-use FURSs on the market, with different characteristics, including 


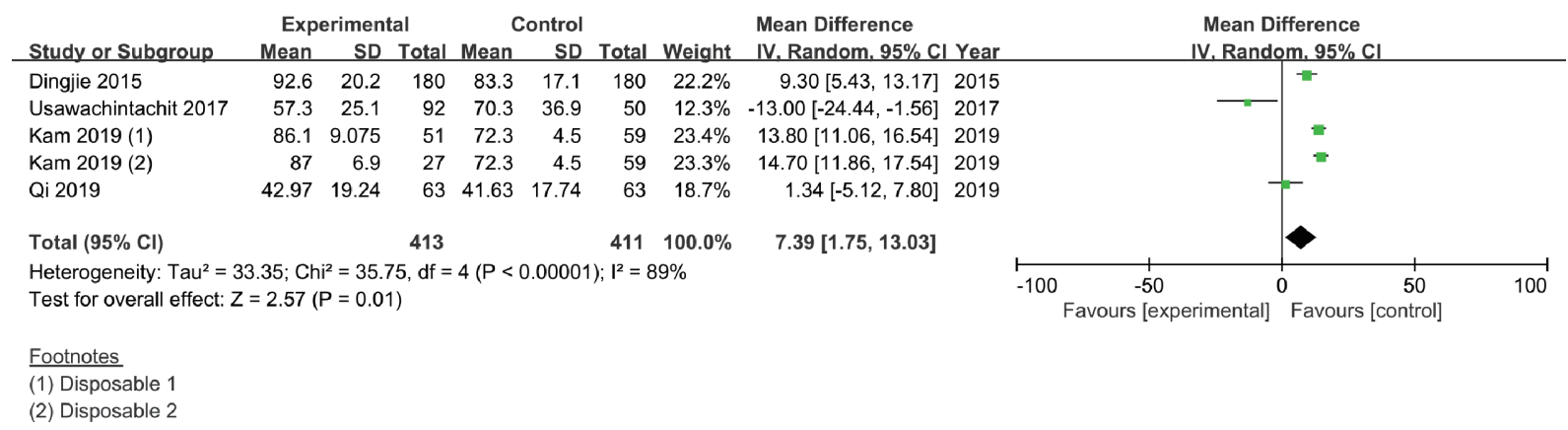

Figure 2 Forest plots illustrating meta-analysis of mean operative time. Single-use FURS was associated with a longer operative time compared with reusable FURS.

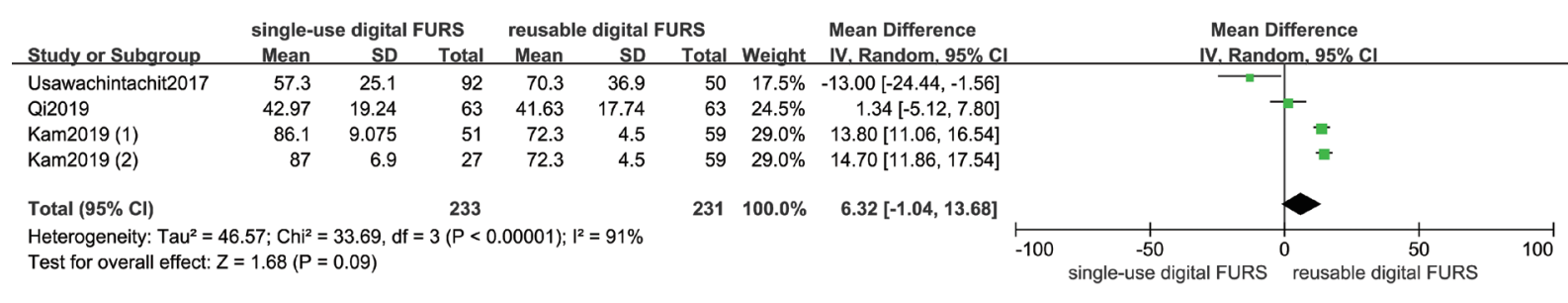

Footnotes

(1) Disposable 1

(2) Disposable 2

Figure 3 Forest plots illustrating meta-analysis of mean operative time. No significant difference between the single-use digital FURS and reusable FURS with regard to operative time.

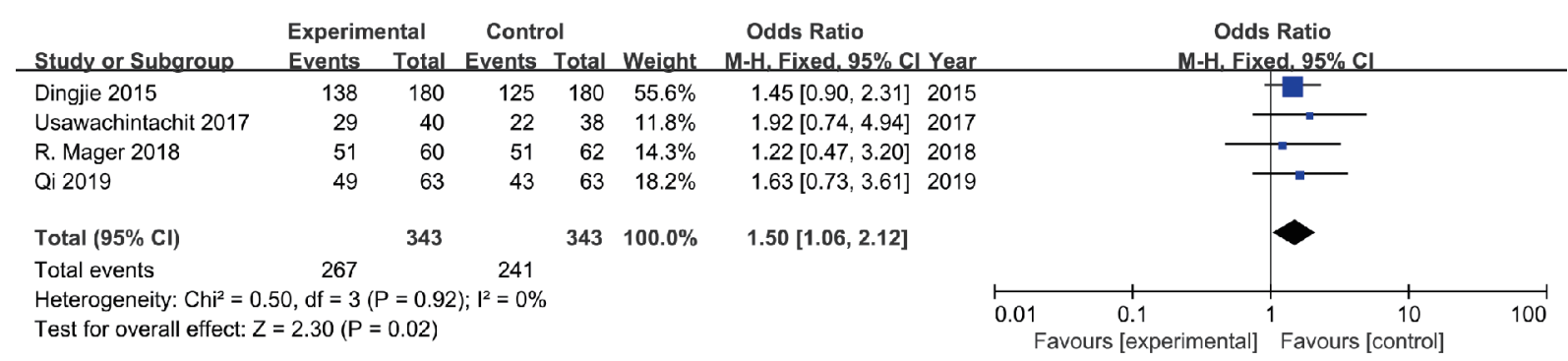

Figure 4 Forest plots illustrating meta-analysis of stone-free rate. The stone-free rate of single-use FURS was superior to that of reusable FURS.

LithoVue, PolyScope, PU3022, Semi-Flex Scope Yc-FR-A and so on (24).

Emiliani et al. published a review of the disposable FURS in 2016 and analysed the characteristics, merits and disadvantages of disposable FURS (24). LithoVue, which is called "the actual single-use FURS", was introduced in Europe in 2015 and launched in the United States in 2016 with the purpose of reducing expenses associated with reusable FURS (25). Benchtop, porcine and cadaveric studies demonstrated that LithoVue is not inferior to reusable FURS when image quality and manoeuvrability were compared $(26,27)$.

The PolyScope is fiberoptic and composed of a single-use flexible catheter (26). It has a modular design to facilitate the repair or disposal of individual components, and the scope contains 10,000-pixel fiberoptic bundles (28). An important limitation of PolyScope is its unidirectional deflection of up to $180^{\circ}$. Gu et al. analysed the clinical performance 
A

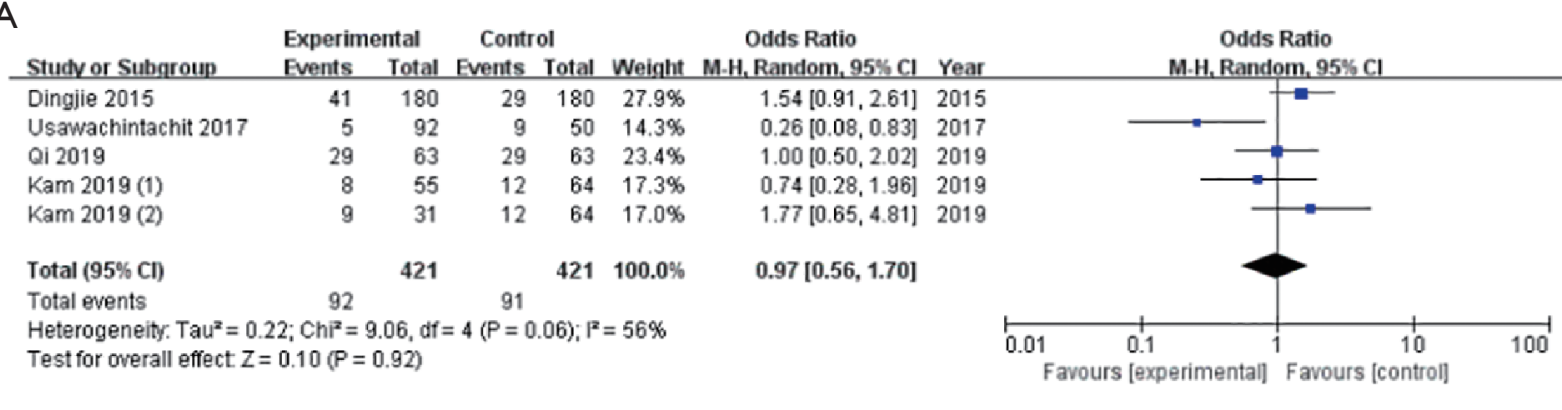

\section{Footnotes \\ (1) Disposable 1 \\ (2) Disposable 2}

B

\begin{tabular}{|c|c|c|c|c|c|c|c|c|c|c|c|}
\hline \multirow[b]{2}{*}{ Study or Subgroup } & \multicolumn{2}{|c|}{ Experimental } & \multicolumn{2}{|c|}{ Control } & \multicolumn{3}{|c|}{ Odds Ratio } & \multirow{2}{*}{\multicolumn{4}{|c|}{$\begin{array}{c}\text { Odds Ratio } \\
\text { M-H, Fixed, 95\% Cl }\end{array}$}} \\
\hline & Events & Total & Events & Total & Weight & M-H, Fixed, $95 \% \mathrm{Cl}$ & Year & & & & \\
\hline Usawachintachit 2017 & 0 & 92 & 1 & 50 & $51.7 \%$ & $0.18[0.01,4.46]$ & 2017 & $\longleftarrow$ & & & \\
\hline Kam 2019 (1) & 1 & 31 & 0 & 64 & $8.4 \%$ & $6.34[0.25,160.29]$ & 2019 & & & & \\
\hline QI 2019 & 0 & 63 & 1 & 63 & $39.9 \%$ & $0.33[0.01,8.21]$ & 2019 & 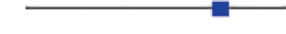 & & & \\
\hline Kam 2019 (2) & 0 & 55 & 0 & 64 & & Not estimable & 2019 & & & & \\
\hline Total $(95 \% \mathrm{Cl})$ & & 241 & & 241 & $100.0 \%$ & $0.76[0.16,3.57]$ & & & & & \\
\hline Total events & 1 & & 2 & & & & & & & & \\
\hline $\begin{array}{l}\text { Heterogeneity: } \mathrm{Ch}^{2}=2 \\
\text { Test for owerall effect: } Z\end{array}$ & $\begin{array}{l}0, d f=2( \\
0.35(P=\end{array}$ & $\begin{array}{l}=0.26) \\
0.73)\end{array}$ & $F^{2}=26 \%$ & & & & & $\begin{array}{ll}0.01 & 0.1 \\
& \text { Favours [experimental] }\end{array}$ & 1 Favours ic & $\begin{array}{l}10 \\
10 \\
\text { control] }\end{array}$ & $100^{\circ}$ \\
\hline
\end{tabular}

\section{Footnotes}

(1) Disposable 2

(2) Disposable 1

C

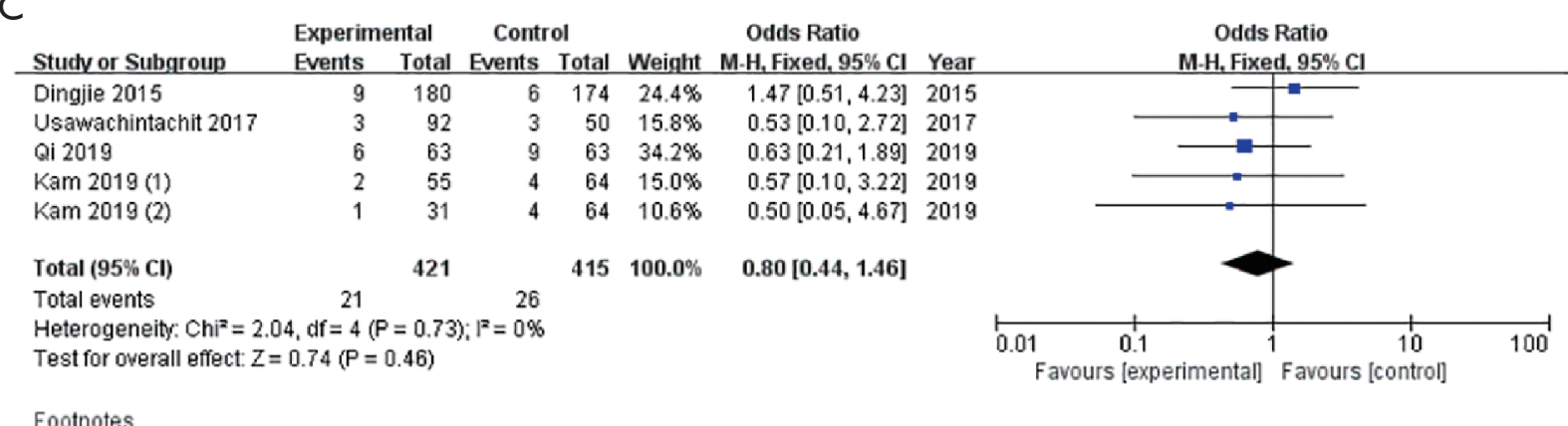

Footnotes

(1) Disposable 1

(2) Disposable 2

D

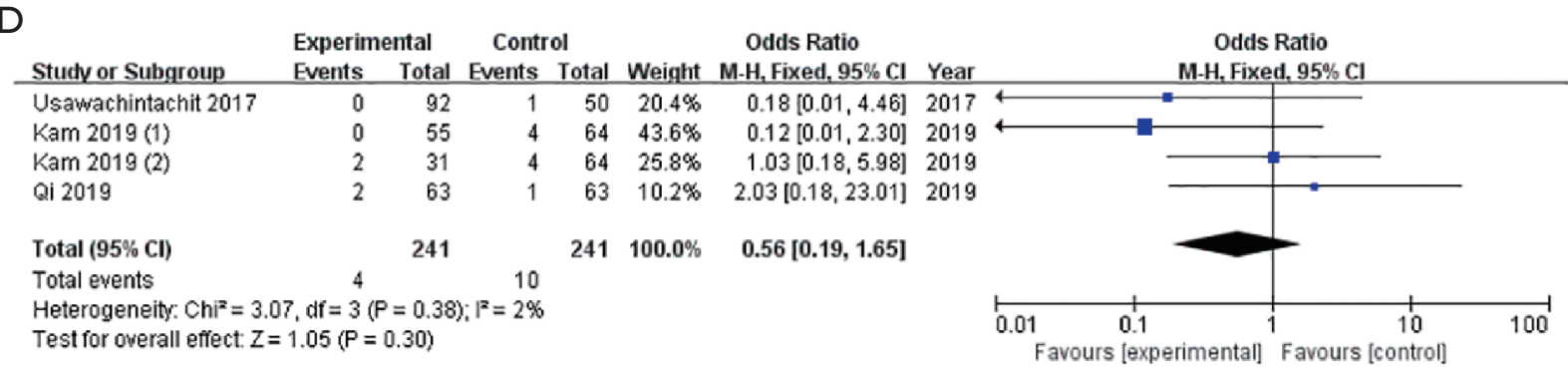

\section{Footnotes}

(1) Disposable 1

(2) Disposable 2

Figure 5 Forest plots illustrating meta-analysis of (A) perioperative complications (B) acute renal injury (C) urinary tract infection (D) stent migration. No significant difference between the single-use digital FURS and reusable FURS with regard to perioperative complications. 


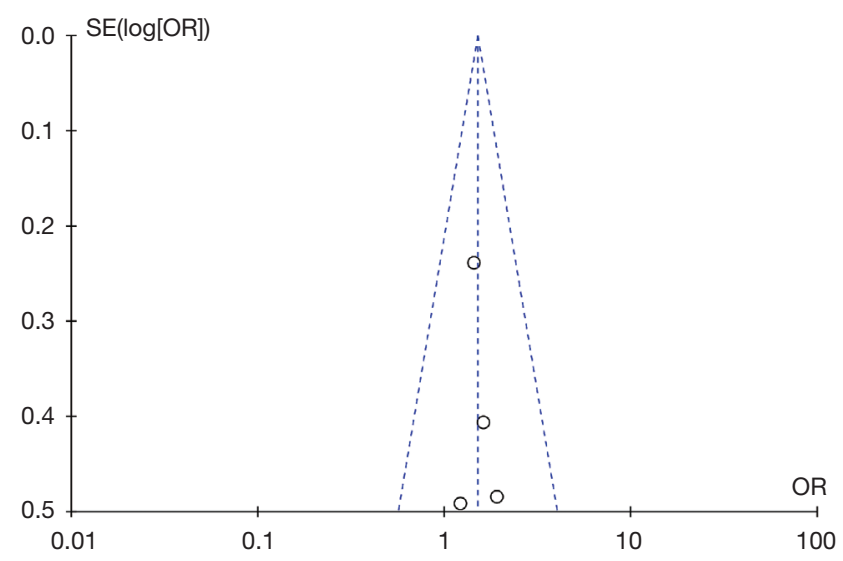

Figure 6 Funnel plot of comparison regarding stone-free rate.

of PolyScope in a series of 86 patients and reported a SFR of $89.5 \%$ and an acceptable safety profile (29).

Uscope PU3022, which is the latest Uscope version, has the same degree of deflection and working channel position as LithoVue. Compared with LithoVue, Pusen's scopes are almost $50 \%$ lighter. Most of the previous studies were focused on physical characteristics. Deininger et al. investigated flow rate, deflection, illumination, and intrapelvic pressure on two single-use and one reusable FURS in a porcine kidney model (15). Higher flow rates were observed in single-use FURS, which was considered to be associated with higher intrapelvic pressure.

It has been reported that the complication rate of FURS is approximately $10-15 \%$ (30). With the purpose of evaluating the clinical outcomes with a Chinese disposable FURS (YouCare Tech), a prospective study was conducted in a single centre in China by Wang et al. with a total of 653 patients involved in the trial. The results showed that the median operative time was $52 \mathrm{~min}$, the overall SFR was $95.2 \%$, and the complications, such as gross haematuria, were mild (30). The discordance of the assessment of SFR resulted in the different results of the SFR. Mager et al. reported a higher SFR in the trial group than in the control group ( $85 \%$ vs. $82 \%$ ) (7). However, in MU's study, the SFR of single-use FURS was $60 \%$ (31). Bader et al. examined the clinical outcome in patients undergoing ureteronephroscopy by PolyScope, and the resulting SFR was $87.5 \%$ (19). The case-control study performed by Usawachintachit et al. found that the overall mean procedure time of single-use FURS was 10 min shorter than that of reusable FURS (19). Conversely, Kam et al. found that single-use FURS was
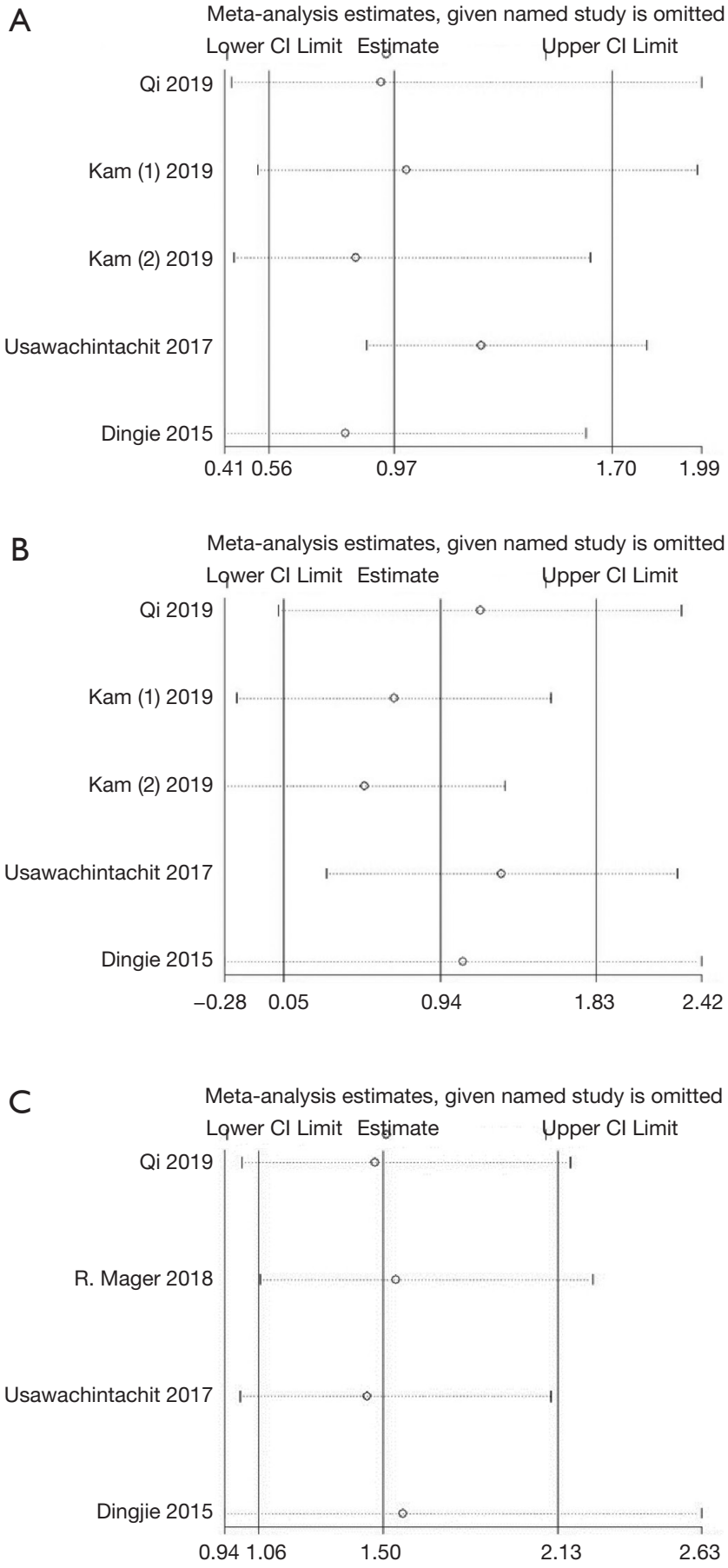

Figure 7 Sensitivity analysis of comparison regarding (A) operative time, (B) stone-free rate and (C) perioperative complications.

10 min longer than that of the reusable FURS group (14). However, JK thought that the lower image quality associated with single-use FURS was responsible for this 
difference, although the operative duration is closely linked with the experience of the surgeon (14). A systematic search was performed for studies evaluating single-use FURS in the setting of urinary tract stone disease by NFD in 2018 (16). The studies consisted of one RCT, three single-centre prospective studies, two multi-institutional prospective studies, one cadaveric feasibility study, one porcine feasibility study and three comparative benchtop studies. The mechanical, optical and irrigation properties of single-use FURS were investigated, and the clinical effectiveness of different single-use FURSs was compared with that of reusable FURSs. The study showed that there were no significant differences in procedure duration, stone clearance or complication rates when single-use FURS and reusable FURS were compared. However, in our study, we excluded cadaveric studies and porcine studies.

Many cost-effectiveness analyses are performed that seem to indicate a lack of economic disadvantage in the employment of single-use FURS, particularly in lowvolume centres. A single-centre retrospective analysis was conducted to compare the actual cost of reusable FURS with LithoVue in 2017 by Ozimek et al. (32), who concluded that, for high volume centres, it is a noneconomic option to shift from reusable FURS to disposable FURS. A systematic literature review on single-use FURS performance was performed in 2020 by Ventimiglia et al. (33), who introduced the available and marketed single-use FURS, the advantages and drawbacks of single-use FURS and the costeffectiveness of single-use FURS. Thus, the wide adoption of single-use FURS is restricted because of its high cost and substantial lack of evidence.

There are several potential limitations to this metaanalysis that should be considered. First, only 5 trials met the inclusion criteria, and among the 5 studies, only 2 were RCTs, while the others were cohort studies or case-control studies. Cohort data are associated with bias caused by patient selection. Second, studies with a small sample size were more likely to overestimate the treatment effect than those with larger sample sizes. Third, SFR was assessed by different methods. These limitations may complicate the interpretation of our findings.

\section{Conclusions}

In conclusion, the findings of the present meta-analysis indicated that single-use FURS had a higher SFR but longer operative time in comparison with reusable FURS.
The perioperative complication rates between the two groups were comparable. Therefore, we propose that single-use FURS may be an alternative therapy to reusable FURS, with acceptable efficacy and complication rates for renal stones. However, considering the potential limitations of this study, further large scale, well performed RCTs are required to verify our findings.

\section{Acknowledgments}

We would like to thank all authors of each included study. Funding: This work was supported by the National Science Foundation of Hunan province, China (2017JJ2395 to YL).

\section{Footnote}

Reporting Checklist: The authors have completed the PRISMA reporting checklist. Available at http://dx.doi. org/10.21037/tau-20-1009

Peer Review File: Available at http://dx.doi.org/10.21037/ tau-20-1009

Conflicts of Interest: All authors have completed the ICMJE uniform disclosure form (available at http://dx.doi. org/10.21037/tau-20-1009). The authors have no other conflicts of interest to declare.

Ethical Statement: The authors are accountable for all aspects of the work in ensuring that questions related to the accuracy or integrity of any part of the work are appropriately investigated and resolved.

Open Access Statement: This is an Open Access article distributed in accordance with the Creative Commons Attribution-NonCommercial-NoDerivs 4.0 International License (CC BY-NC-ND 4.0), which permits the noncommercial replication and distribution of the article with the strict proviso that no changes or edits are made and the original work is properly cited (including links to both the formal publication through the relevant DOI and the license). See: https://creativecommons.org/licenses/by-nc$\mathrm{nd} / 4.0 \%$.

\section{References}

1. Chen J, Zhang D, Ji MF, et al. Activation of liver X 
receptor suppresses osteopontin expression and ameliorates nephrolithiasis. J Cell Physiol 2019;234:14109-22.

2. Alelign T, Petros B. Kidney Stone Disease: An Update on Current Concepts. Adv Urol 2018;2018:3068365.

3. Al-Qahtani SM, Gil-Deiz-de-Medina S, Traxer O. Predictors of clinical outcomes of flexible ureterorenoscopy with holmium laser for renal stone greater than $2 \mathrm{~cm}$. Adv Urol 2012;2012:543537.

4. Türk C, Petř́k A, Sarica K, et al. EAU Guidelines on Interventional Treatment for Urolithiasis. Eur Urol 2016;69:475-82.

5. Heers H, Turney BW. Trends in urological stone disease: a 5-year update of hospital episode statistics. BJU Int 2016;118:785-9.

6. Marshall VF. Fiber optics in urology. J Urol 1964;91:110-4.

7. Mager R, Kurosch M, Höfner T, et al. Clinical outcomes and costs of reusable and single-use flexible ureterorenoscopes: a prospective cohort study. Urolithiasis 2018;46:587-93

8. Bryniarski P, Paradysz A, Zyczkowski M, et al. A randomized controlled study to analyze the safety and efficacy of percutaneous nephrolithotripsy and retrograde intrarenal surgery in the management of renal stones more than $2 \mathrm{~cm}$ in diameter. J Endourol 2012;26:52-7.

9. Shah K, Monga M, Knudsen B. Prospective Randomized Trial Comparing 2 Flexible Digital Ureteroscopes: ACMI/ Olympus Invisio DUR-D and Olympus URF-V. Urology 2015;85:1267-71.

10. Marchini GS, Batagello CA, Monga M, et al. In Vitro Evaluation of Single-Use Digital Flexible Ureteroscopes: A Practical Comparison for a Patient-Centered Approach. J Endourol 2018;32:184-91.

11. Bagley DH. Flexible ureteropyeloscopy with modular, "disposable" endoscope. Urology 1987;29:296-300.

12. Bourdoumis A, El Howairis MEF, Stephen B, et al. Ex vivo Comparison of 2 Disposable versus a State-of-the-Art Reusable Ureterorenoscope. Urol Int 2020;104:437-44.

13. Dale J, Kaplan AG, Radvak D, et al. Evaluation of a Novel Single-Use Flexible Ureteroscope. J Endourol 2017. [Epub ahead of print].

14. Kam J, Yuminaga Y, Beattie K, et al. Single use versus reusable digital flexible ureteroscopes: A prospective comparative study. Int J Urol 2019;26:999-1005.

15. Deininger S, Haberstock L, Kruck S, et al. Single-use versus reusable ureterorenoscopes for retrograde intrarenal surgery (RIRS): systematic comparative analysis of physical and optical properties in three different devices. World J
Urol 2018;36:2059-63.

16. Davis NF, Quinlan MR, Browne C, et al. Single-use flexible ureteropyeloscopy: a systematic review. World J Urol 2018;36:529-36.

17. Liberati A, Altman DG, Tetzlaff J, et al. The PRISMA statement for reporting systematic reviews and metaanalyses of studies that evaluate healthcare interventions: explanation and elaboration. BMJ 2009;339:b2700.

18. Ding J, Xu D, Cao Q, et al. Comparing the Efficacy of a Multimodular Flexible Ureteroscope With Its Conventional Counterpart in the Management of Renal Stones. Urology 2015;86:224-9.

19. Usawachintachit M, Isaacson DS, Taguchi K, et al. A Prospective Case-Control Study Comparing LithoVue, a Single-Use, Flexible Disposable Ureteroscope, with Flexible, Reusable Fiberoptic Ureteroscopes. J Endourol 2017;31:468-75.

20. Qi S, Yang E, Bao J, et al. Single-Use Versus Reusable Digital Flexible Ureteroscopes for the Treatment of Renal Calculi: A Prospective Multicenter Randomized Controlled Trial. J Endourol 2020;34:18-24.

21. Bell JR, Penniston KL, Best SL, et al. Prospective evaluation of flexible ureteroscopes with a novel evaluation tool. Can J Urol 2017;24:9004-10.

22. Doizi S, Kamphuis G, Giusti G et al. First clinical evaluation of a new single-use flexible ureteroscope (LithoVueTM): a European prospective multicentric feasibility study. World J Urol 2017;35:809-18.

23. Somani BK, Al-Qahtani SM, de Medina SD, Traxer O. Outcomes of flexible ureterorenoscopy and laser fragmentation for renal stones: comparison between digital and conventional ureteroscope. Urology 2013;82:1017-9.

24. Emiliani E, Traxer O. Single use and disposable flexible ureteroscopes. Curr Opin Urol 2017;27:176-81.

25. Cho SY, Lee JY, Shin DG, et al. Evaluation of Performance Parameters of the Disposable Flexible Ureterorenoscope (LITHOVUE) in Patients with Renal Stones: A Prospective, Observational, Single-arm, Multicenter Study. Sci Rep 2018;8:9795.

26. Xu K, Ding J, Shi B, et al. Flexible ureteroscopic holmium laser lithotripsy with PolyScope for senile patients with renal calculi. Exp Ther Med 2018;16:1723-8.

27. Proietti S, Dragos L, Molina W, et al. Comparison of New Single-Use Digital Flexible Ureteroscope Versus Nondisposable Fiber Optic and Digital Ureteroscope in a Cadaveric Model. J Endourol 2016;30:655-9.

28. Dessyn JF, Balssa L, Chabannes E, et al. Flexible Ureterorenoscopy for Renal and Proximal Ureteral 
Stone in Patients with Previous Ureteral Stenting: Impact on Stone-Free Rate and Morbidity. J Endourol 2016;30:1084-8.

29. Gu SP, Huang YT, You ZY, et al. Clinical effectiveness of the PolyScope ${ }^{\mathrm{TM}}$ endoscope system combined with holmium laser lithotripsy in the treatment of upper urinary calculi with a diameter of less than $2 \mathrm{~cm}$. Exp Ther Med 2013;6:591-5.

30. Wang F, Yang Y, Chen H, et al. The application of a single-use fiberoptic flexible ureteroscope for the management of upper urinary calculi. Int Urol Nephrol 2018;50:1235-41.

Cite this article as: Li Y, Chen J, Zhu Z, Zeng H, Zeng F, Chen Z, Yang Z, Cui Y, Chen H, Li Y. Comparison of single-use and reusable flexible ureteroscope for renal stone management: a pooled analysis of 772 patients. Transl Androl Urol 2021;10(1):483-493. doi: 10.21037/tau-20-1009
31. Bader MJ, Gratzke C, Walther S, et al. The PolyScope: a modular design, semidisposable flexible ureterorenoscope system. J Endourol 2010;24:1061-6.

32. Ozimek T, Schneider MH, Hupe MC, et al. Retrospective Cost Analysis of a Single-Center Reusable Flexible Ureterorenoscopy Program: A Comparative Cost Simulation of Disposable fURS as an Alternative. J Endourol 2017;31:1226-30.

33. Ventimiglia E, Somani BK, Traxer O. Flexible ureteroscopy: reuse? Or is single use the new direction? Curr Opin Urol 2020;30:113-9. 
Supplementary

Table S1 The quality of evidence of the included non-RCT trails

\begin{tabular}{|c|c|c|c|c|c|c|c|c|c|c|}
\hline Study & \multicolumn{3}{|c|}{ Selection } & \multicolumn{3}{|c|}{ Comparability } & \multicolumn{3}{|c|}{ Exposure } & Scores \\
\hline Kam & is & & & ist & is & & is & it & is & 6 \\
\hline R.mager & is & is & & it & 岤 & & 聍 & 岤 & & 6 \\
\hline Usawachintachit & is & is & & tht & $i s$ & & 放弥 & is & & 7 \\
\hline
\end{tabular}

a:adequate case definition; b: representativeness of the cases; c: selection of controls; d:definition of controls; e:study controls for the most important factor; f: study controls for any additional factor; g: ascertainment of exposure; h: some methods of ascertainment for cases and controls; i: non-response rate

Table S2 The quality of evidence of the RCT trails

\begin{tabular}{|c|c|c|c|c|c|}
\hline Study & Generation of random Sequences & Randomization concealment & Blinding & Withdrawals and dropouts & Scores \\
\hline Ding jie & 论坛 & 㧒 & 岤 & 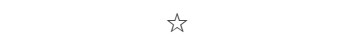 & 5 \\
\hline
\end{tabular}

